\title{
When the world becomes too real: a Bayesian explanation of autistic perception
}

\author{
Elizabeth Pellicano ${ }^{*} \&$ David Burr ${ }^{2}$ \\ 'Centre for Research in Autism and Education (CRAE), Institute of Education, University of \\ London, UK; ${ }^{2}$ Department of Psychology, University of Florence, Florence, Italy
}

Abstract word count: 118 words (max. 120 words)

Manuscript word count: 3287 (main text) +400 (Box I) $=3687$ (max. 3700 words)

*Corresponding author:

Dr Liz Pellicano

Centre for Research in Autism and Education

Department of Psychology and Human Development

Institute of Education

25 Woburn Square

Tel: +44 (0)207 33I 5I40

London WCIH OAA

Email: I.pellicano@ioe.ac.uk 


\begin{abstract}
Our perceptual experience is influenced both by incoming sensory information and prior knowledge about the world: a concept recently formalized within Bayesian decision theory. We propose that Bayesian models can be applied to autism - a neurodevelopmental condition with atypicalities in sensation and perception - to pinpoint fundamental differences in perceptual mechanisms. We suggest specifically that attenuated Bayesian priors - hypo-priors - may be responsible for the unique perceptual experience of autistic people, leading to a tendency to perceive the world more accurately rather than modulated by prior experience. In this account, we consider how hypo-priors might explain the key features of autism - the broad range of sensory and other non-social atypicalities - in addition to the phenomenological differences in autistic perception.
\end{abstract}


Autism is a heritable, lifelong neurodevelopmental condition that has its most striking effects on social communication - the so-called social symptoms. Yet the condition is also defined by a less well-researched range of non-social symptoms. These symptoms present throughout development, are prevalent in autistic individuals regardless of intellectual ability, and vary widely from an intense desire for sameness (like following rigid routines) and sensory atypicalities (such as extreme sensitivity to florescent lighting or to the sound of the school bell) to remarkable talents (such as an excellent eye for detail).

These symptoms feature prominently in the draft changes to the forthcoming diagnostic guidelines for autism [I] but the range and idiosyncrasy of sensory atypicalities in particular still represent some of the most puzzling features of autism. They include not only hypersensitivity to incoming stimuli but also hyposensitivity to stimuli and sensory seeking behaviours such as attraction to light, intense looking at objects and fascination with brightly coloured objects $[2$, 3]. Indeed, they often oscillate between these states within the same individual. They can also have catastrophic effects on the lives of autistic people. As Donna Williams reports first hand: "the sensory overload caused by bright lights, fluorescent lights, colours, and patterns makes the body react as if being attacked or bombarded, resulting in such physical symptoms as headaches, anxiety, panic attacks or aggression" [4, p. 43].

There has been renewed research interest in these sensory symptoms, prompted in part by the possibility that the non-social symptoms of autism might be attributable to fundamental differences in sensation and perception [5-10]. In this article, we propose a new account of the sensory and other non-social symptoms of autism, which we believe provides a parsimonious explanation for such atypicalities; that people with autism see the world more accurately - as it really is - as a consequence of being less biased by prior experiences. 
We start with the suggestion that it is not sensory processing itself that is different in autism, but the interpretation of sensory input to yield percepts. We further propose that Bayesian decision theory, a principled description of the processes that enable observers to derive the most probable interpretations of their environment (see Box I), provides a powerful tool to study the mechanisms underlying the diverse range of non-social features in autism. Such computational methods should formalise the process of generating experimentally testable hypotheses about the underlying functional atypicalities in autistic perception. Specifically we suggest that atypicalities exist at the level of our internal, working models of the world - priors in Bayesian terms - and that these lead to characteristic differences in autistic sensation and perception.

\section{Perceptual processing in autism}

It has long been known that perceptual processing is unusual in autism. Early studies reported autistic exceptional performance on the Embedded Figures Test, finding hidden figures (e.g., triangle) within larger meaningful drawings (e.g., pram) [II]. Other studies have shown less susceptibility to visual illusions [12], the prevalence of absolute pitch [13], enhanced performance on visual search tasks $[14,15]$ and superior visual discrimination $[5,16]$. These initial studies spawned a raft of further investigations [see 17], generally revealing atypicalities in the perception of characteristically non-social stimuli, such as chromatic stimuli [18], isolated tones [19], coherently moving dots [20] and complex objects [2I], as well as social stimuli, including faces [2I, 22], eye-gaze direction [23, 24], biological motion [25, 26], and speech [27].

There have been several influential accounts of the non-social symptoms and perceptual processing differences in autism, which each differ with regard to the precise nature of the 
atypicality. Frith and Happé's weak central coherence hypothesis (1989; Happé \& Frith, 2006) was the first to suggest that the non-social symptoms in autism - the weaknesses and the strengths - could be explained by a domain-general processing style that afforded "privileged access to parts and details" (Frith \& Happé, 1994, p. 122) and resulted in difficulties processing information in context. Later, they suggested that problems in top-down modulation could lead to hypersensitivity to sensory stimuli in autism ("naming the pitch of the 'pop' of a cork") and that a detail-focused processing style caused the characteristic "insistence on sameness" (Happé \& Frith, 2006).

Others have posited alternative accounts, which move beyond the focus on local-global processing in autism, and place autistic differences squarely in the realm of perception. Plaisted [9] proposed that autistic individuals' perceptual atypicalities were due to enhanced discrimination, possibly from enhanced lateral inhibition in perception. Similarly, Mottron et al. [8] (2006) suggest within their "Enhanced Perceptual Functioning" (EPF) account that autistic perception is characterized by enhancements in bottom-up, feedforward perceptual operations. These authors [28] further suggest that autistic perception is autonomous from higher-level, top-down influences and may involve a one-to-one or veridical mapping process. On this account, hypersensitivity in autism results from an imbalance in inhibitory and excitatory connectivity between local neural networks in sensory regions [see also $5,9,10,29,30]$.

Despite their prominence in the autism field, the impact of these accounts has been limited both by the lack of data demonstrating an empirical link between theoretical constructs - such as "top-down control" - and autistic sensory and other non-social atypicalities and, in 
some cases, by their overly descriptive nature, failing to fully specify the underlying (altered) mechanisms, that is, the nature of the computations.

Moreover, these accounts focus predominantly on enhancements in sensation and perception (hypersensitivity) in autism. Yet the nature and degree of sensory atypicalities in autism - hypersensitivity, hyposensitivity and sensory seeking behaviours - vary enormously and reportedly fluctuate even within the same individuals. These sensory but also the other nonsocial features of autism present a serious challenge for current explanations of autism. Furthermore, such theories also have difficulty accounting for an apparent paradox, first noted by Kanner [3I] in which "the child himself can happily make as great a noise as any that he dreads and move objects about to his heart's content" (p. 245), despite being distressed by incoming noises or movements. What is particularly unsettling for autistic individuals is therefore the unexpected and unpredictable nature of external events. We suggest that understanding how perceptual systems deal with uncertainty is key to explaining atypicalities in autistic sensation and perception.

\section{Perception as inference}

Recognizing that retinal images are inherently ambiguous, Helmholtz [32] suggested that perception is a process of unconscious inference: automatic and unconscious "best guesses" about the structure of the world, consistent with both the retinal images and past experience. Gregory [33] advanced a similar idea, that perception is an active process of formulating and testing hypotheses about the structure of the world. He richly illustrated his idea with vivid examples of visual illusions, such as perceiving a physical hollow mask as a convex face. Seeing familiar shapes or images in the clouds, or the "ghostly" surfaces of the 
Kanizsa triangle, are examples of the brain postulating the most likely interpretation for noisy, ambiguous sensory signals (see Figure I).

Bayesian statistical decision theory, a principled method of optimal reasoning under uncertainty, formalizes Helmoltz's and Gregory's notions of perception as unconscious inference [34-37]. Box I lays out the basic principles of Bayesian inverse inference, illustrated by the specific example of Figure 2 . The simple image of the figure is consistent with many different physical shapes, depending on viewpoint, described by the likelihood function; but some are more prevalent in normal viewing than others, leading to a probability distribution referred to as the prior. The prior is combined with the likelihood to yield the posterior probability distribution, narrower than either the prior or the likelihood, whose maximum is taken as the statistically best estimate of the shape creating that image. If the prior is appropriate, the Bayesian framework provides the most efficient method to infer the 3D shape corresponding to the simple 2D line drawing.

In the real world, many other forms of knowledge are available as priors to aid disambiguation, such as the fact that light more probably comes from above, so shading can provide useful information [38]. Priors can explain many visual illusions. For example, the Kanizsa triangle (see Figure I) is equally consistent with a continuous white triangle superimposed over three regular black circles, or three unlikely "pac-men" arranged symmetrically to face each other: the natural statistics of the world makes the single triangle more probable. Similarly, an a priori preference for slow speeds can aid disambiguation of motion direction, but is also consistent with many illusory perceptions of incorrect velocity [39]. Consistent with Gregory's view, these authors regard illusions not as perceptual errors or 
“sloppy computations", but are a consequence of statistically optimal computations that are functionally beneficial in the real world [39].

In general, priors improve the efficiency of computations by reducing overall noise or error. Even when images are not ambiguous, this can be advantageous. For example, psychophysical judgments of almost all quantities - length, duration, number, color, weight, force - show the tendency to gravitate towards mean magnitude [40]. This fact has been well known for at least 100 years, but still not well understood. Recently Jazayeri and Shadlen [4I] suggested that central tendency may represent another statistically optimal strategy, incorporating prior knowledge of the statistics of the environment in psychophysical judgments. They suggest that the mean duration (or length, color, or weight) of the recent history acts as a prior, biasing judgments towards the mean. Although judgments are biased ("inaccurate”), reliability is improved and overall error-rate is reduced. Interestingly, this theoretical approach (supported by clear data [4I, 42]) suggests that priors do not need to be learned over a lifetime, but can be modulated over a relatively short timescale, in the order of minutes.

The above examples are intentionally simple, with only a few relevant variables such as curvature and slant, but the principles readily extend to high-dimensional space. Furthermore, advances in computational neuroscience are beginning to demonstrate how such probabilistic inference is instantiated in the brain. Some have shown that populations of neurons can code entire probability distributions relating to a stimulus and also the degree of uncertainty for computations like cue combination [43]. Others have suggested that probabilistic perception and learning should be better implemented with sampling-based approaches, whereby single neurons map on to inferred variables and uncertainty is represented by the variability of neural activity patterns [44]. Intriguingly, these authors have proposed that a priori beliefs about the 
world (priors) reside in spontaneous cortical activity (activity in the absence of sensory stimulation) - activity which is thought to be atypical in autism [45].

\section{Autistic perception within a Bayesian framework}

We suggest that the Bayesian framework could be particularly useful for deriving testable hypotheses about functional atypicalities in autistic perception. Specifically, we propose that altered autistic perception results from atypicalities at the level of the prior - either in its construction or in combining appropriately with sensory information - yielding unusually attenuated priors or hypo-priors (see Figure 2). The suggestion here is not that individuals with autism have no priors, rather that their priors are broader. If true, we would expect that fewer internal constraints on perception - hypo-priors - should have substantial effects on autistic individuals' perceptual experiences.

One prediction is that hypo-priors should result in more "accurate" perception. As mentioned above, Bayesian priors sacrifice accuracy (understood as average closeness to physical reality) for improved precision (reliability), resulting in an overall reduction of error. Under many conditions, strong (narrow) priors can bias perception towards the prior, away from the maximum likelihood, which is based only on sensory information. Hypo-priors in autism should distort sensory signals less, consistent with the often-reported superior performance of autistic individuals [46-50]. They are, for example, less susceptible to illusions such as the Kanizsa, Titchener, Poggendorf and Shepard "table-illusion" [7, I2] (see Figure I). Individuals with autism are also better at copying impossible figures [5I] and are more accurate when asked to reproduce a slanted circle (ellipse) in the absence of perspective cues [52]. In all these somewhat artificial tasks, priors should actually impede performance. 
A second prediction is that hypo-priors should impede performance in situations where priors help resolve ambiguity. For example, cast-shadows provided useful information about shape, if interpreted appropriately [38], and indeed make objects more recognizable for typical individuals. For autistic children, however, cast shadows hinder recognition [53] - a finding that is consistent with the suggestion that autistic children make less use of prior information to interpret shadows appropriately. In this case, cast shadows just add to the perceptual noisiness of an image [38].

A third, and less obvious, prediction is that hypo-priors in autism could cause the oftenreported sense of being overwhelmed by sensory information. Mukchopadhyay describes the experience of seeing everything afresh, rather than mediated by prior knowledge and expectation: "I began to fear all those unknown paths, clothes, shoes, chairs and strange human voices. Each one challenged me by putting me in front of a new situation for me to face and understand..." [3]. There are two ways that hypo-priors may lead to this form of phenomenon. Priors - such as the example of central tendency - smooth variations in sensory input, often caused by measurement error rather than real variability: hypo-priors would result in more unexpected variability, even in constant stimuli. Alternatively, they could affect the learning process itself. Recent accounts show how knowledge of underlying image statistics is fundamental for learning [44] - as it is for perception. Attenuated priors could result in reduced capacity for generalization during learning, akin to what is known as "overfitting" in computer vision, fitting a model to noisy data rather than to the general trend. In both cases, hypo-priors would result in a mismatch between expectations and measurement, which could lead to phenomenological reports like Mukchopadhyay's. 
A Bayesian framework might also help us to interpret findings of reduced adaptation in autism. Adaptation - ubiquitous in perceptual systems - is a rapid form of experiencedependent plasticity where sensory experience affects the response properties of neurons and, ultimately, perception [54]. It is generally accepted that adaptation serves to auto-calibrate perceptual systems to their environment $[55,56]$. The effects of adaptation in autism have been investigated with the face aftereffect, where prolonged exposure to a specific facial identity biases subsequent perception away from that identity [57]. Children with autism showed significantly less adaptation than typical children. Critically, their perception was more accurate, in that the target face corresponded better to physical reality than to expectations. Subsequent studies have demonstrated diminished adaptation in autism for other sensory modalities, including touch [58], and for relatives of autistic children [59].

Adaptation does not always cause negative effects. In a recent study, Chopin and Mamassian [60] have shown that the effects of adaptation depend on when in the past the adapting stimuli occur. Recent adaptors affect the current percept negatively, biasing - for example - the perceived tilt in the direction opposite to the adaptors; adapting stimuli further in the past act in the opposite way, biasing tilt in the same direction as the adaptors. These results have very important implication for Bayesian explanations of adaptation \{Stocker, 2006 \#55; Clifford, 2012 \#2 I3\}. Self-calibration theories of adaptation assume that the brain has some internal model of the expected distribution of response states. Within the Bayesian framework, the positive effects of adaptation of remote stimuli [60] suggest that the brain continually learns and updates the probability distributions of the world, over a moderately short timescale (5-10 minutes): the learnt distributions serve as priors, or standards for selfcalibration. The negative effects of recent stimuli could represent a recalibration of sensory 
resources under the assumption that the recent history of sensory input should conform to the established priors. Clearly, any atypicality at the level of the prior - either in its construction or use as a calibration standard - should clearly impact on the magnitude of adaptation. That is, hypo-priors in autistic perception may lead to difficulties in using information from the remote past to drive expectations about incoming sensory signals.

\section{Hypo-priors may explain many of the non-social symptoms of autism}

Hypo-priors in autism should cause a greater reliance on bottom-up, incoming sensory signals, which could in turn result in enhancement of sensory stimuli more broadly. Enhanced sensations, or "super qualia" [62] are consistent with the often-reported hypersensitivity to sensory information $[4,63]$. Attenuated prior knowledge could also explain the co-occurrence of hyposensitivity and hypersensitivity within the same individuals. Without a template against which to match observed sensory evidence, the individual is less able to anticipate the forthcoming sensory environment in order to resolve perceptual ambiguity. Fewer internal constraints could also lead to a sense of alarm and the often-reported experience of sensory overload. Sensory symptoms in autism would therefore not be due to fundamental differences in sensory processing per se but rather reflect atypicalities in the way that incoming information is interpreted by sensory systems.

Furthermore, since it is assumed by Bayesian theory (see Box I) that priors are altered according to the specific stimuli the individual encounters, the idiosyncratic pattern of sensory seeking (e.g., attraction to spinning objects) and hyper-sensitivity to stimuli (e.g., aversion to vacuum cleaners) is likely to be determined by the amount of and intensity of exposure to particular stimuli in the individual's environment. 
Hypo-priors might also explain why autistic behaviours can be stereotyped and resistant to change. Prior knowledge should aid in the interpretation of predictable sensory events. Sensory experiences that are less constrained by prior knowledge should therefore make it difficult to use knowledge derived from the past to generate predictions about the occurrence of new sensory events. This may shed light on the intense desire for sameness in autism, which may be not a problem with change per se, but in predicting the change. Becoming comfortable with new situations might also require many more exposures to a stimulus or context to overcome the potentially disadvantageous effects of less specific priors.

Well-known repetitive or "stimming" behaviours such as rocking, finger flicking, hand flapping, might also be accounted for by hypo-priors. Less specific priors could result in reduced generalization, which in turn could constrain motor plans to those that are already known. Without the moderating effect of priors, self-generated repetitive behaviours - those over which the individual has full control - might be a means of reducing the uncertainty in the environment.

\section{Conclusion}

In this article we have proposed that the formal, computational principles of a Bayesian framework offer a way forward in identifying the causal mechanisms of altered autistic perception. We have suggested here that autistic people tend to perceive the world more accurately as a consequence of hypo-priors or less bias by prior experience - a notion that fits

well with extant empirical data. Certain aspects of our account have been raised previously [6, 7]. The distinct advantage of our account is that it has greater explanatory power than existing 
theories by providing a unifying explanation of the sensory and other non-social features of autism, as well as atypicalities (both weaknesses and strengths) in autistic perception.

The Bayesian framework also allows for concepts like top-down knowledge and contextual processing to be translated into testable theories about the strength or reliability of priors, and therefore serves as an excellent platform to assess the internal coherence and completeness of these ideas. Indeed, such methods should help to specify the precise nature of the atypicality - whether it lies either in the application of priors or in the learning and generating of new priors, or indeed in both [cf 44]. Future empirical work and computational modelling will no doubt determine its usefulness in elucidating the autistic experience of the world (see Outstanding Questions), and will hopefully lead to suggestions of how they may better cope with it. 


\section{Acknowledgements}

We extremely grateful to Dorothy Bishop, Tom Griffiths, Rebecca Lawson, Kate Plaisted-

Grant, Marc Stears and Catherine Stoodley for helpful discussions and to Colin Clifford, Laurent Mottron and three anonymous reviewers for constructive comments on a previous version of this manuscript. This work was supported by a grant from the UK's Medical Research Council (MR/J0I3 I45/I) and European Union FP7-ERC “STANIB”. Research at CRAE is also supported by The Clothworkers' Foundation and Pears Foundation. 


\section{Glossary}

Adaptation: A dynamic process in which neural sensitivity is continuously recalibrated to "match" the characteristics of the current environment.

Aftereffect: The perceptual distortions that arise following lengthy exposure or adaptation to a stimulus.

Autism: Autism spectrum disorders are a set of common, lifelong neurodevelopmental conditions defined in terms of the presence of difficulties in social communication and social interaction, and a range of restricted, repetitive patterns of behavior, interests, or activities, including sensory sensitivities.

Bayesian inverse inference: A method of statistical inference in which Bayes' rule is used with reference to an unknown variable (see Box 1).

Hypo-priors: A term we use to describe attenuated prior knowledge in autism, which would be represented as a broad prior probability distribution.

Likelihood: the function specifying the probability $p(x j y)$ of observing a particular stimulus $x$ for each possible state of the environment $y$.

Non-social symptoms: The range of autistic symptoms, which include restricted, repetitive patterns of behavior, interests, or activities, and sensory sensitivities, which are relatively non-social in nature and content.

Posterior: the probability distribution $p(y j x)$ produced by probabilistic inference according to a particular probabilistic model of the environment. 
Prior: the probability distribution $\mathrm{p}(\mathrm{y})$ defining the expectation about the environment being in any of its possible states, $y$, before any observation is available. 


\section{Box I. The Bayesian approach to object perception}

The Bayesian framework for perception originates in Helmholtz's [32] notion of perception as "unconscious inference". Helmholtz realised that many images are inherently ambiguous, so prior knowledge is necessary to disambiguate them. Recently, this concept has been formalised by models based on Bayes' formula for inverse inference:

$$
p(S \mid I)=\frac{p(I \mid S) p(S)}{p(I)}
$$

where $S$ is the shape of the object and $I$ the image formed by it. Figure 2 illustrates a simple example. The image $(I)$ in panel $\mathrm{A}$ is ambiguous, consistent with multiple objects and viewpoints, including the three illustrated in the panel: most observers' first impression is a halfpipe (or Tuscan roof tile) viewed from above, but it can be "willed" into other configurations, such as a convex tile. The full range of shapes consistent with the (noisy) measurement of image I is given by the likelihood function (I|S: panel B). To be consistent with the image, the curvature of the physical object must increase as the object surface slant approaches zero (orthogonal to line of sight). However, it can never reach 0 or $\pm \pi / 2$, as neither extreme could produce the two crescents with connecting lines. The object curvature could either be positive (concave), or negative (convex), depending on slant, but could not be positive for negative slant or vice versa. The scatter of probabilities around the functions reflects the noisiness (imprecision) in measuring the image (I). Panel $\mathrm{C}$ shows an example of a typical prior, a distribution of probable shapes. Humans show a preference for the from-above viewpoint, and also for curvatures equal to or smaller than circles. When multiplied by the likelihood, it gives a posterior probability function (panel D), far more constrained than the likelihood. The maximum a posteriori (MAP) is the peak of this function, the best Bayesian guess of the shape to produce the image $\max (p(S \mid))$, falling close to shape $Y$. Note that there is also a small mound corresponding to negative 
curvature, corresponding to the less favoured but possible interpretation, shape $Z$. If the prior were attenuated (more distributed), as in panel E, it would constrain the image less (panel F), leaving $X, Y$ and $Z$ almost equally probable. It is possible that this is the case with autism.

How the prior is generated remains open to question, many believing it develops over the lifespan, and perhaps evolves over generations. However, several recent studies [4I, 42, 60] suggest that even 5-10 minutes of learning can be sufficient to alter the prior. 


\section{Figures}
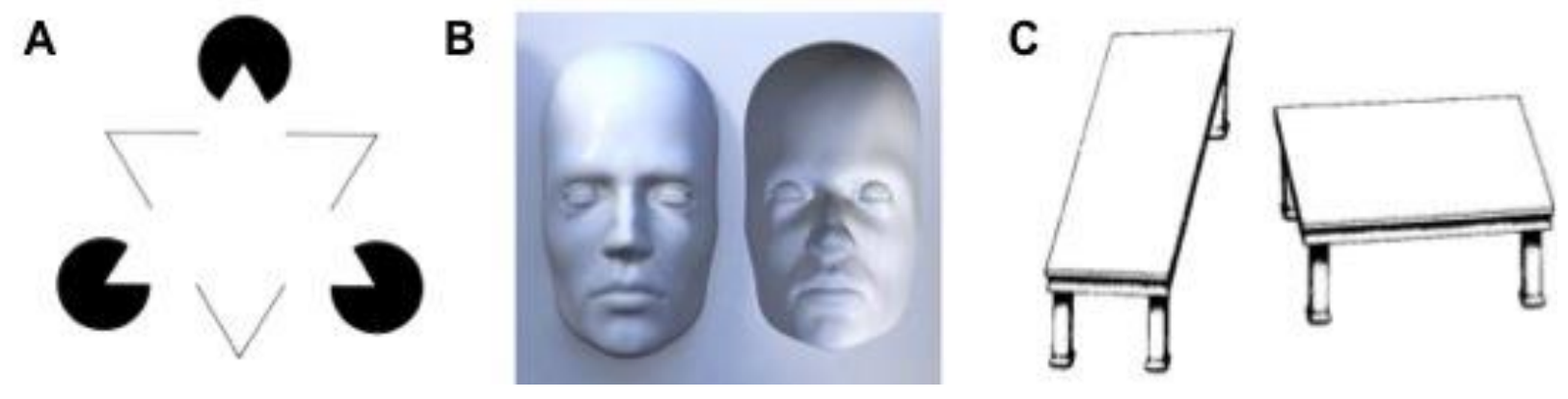

Figure I. Some examples that powerfully demonstrate the importance of prior knowledge in interpreting ambiguous bottom-up information. A. The Kanizsa triangle: three circles with segments missing and a triangle placed directly in front of them. The edges of the triangle are not really there, but would be for the most probable physical interpretation, a white triangle overlaying three regular circles. B. The Hollow-face illusion. A strong bias (or "prior") for natural concave faces offsets competing information (such as shadows) and causes one to perceive a concave, hollow mask (right) as a normal convex face (left). C. Shepard's table illusion. The two-dimensional images of the tabletop parallelograms are in fact identical. However, the image is consistent with many three-dimensional shapes, the most probable being real tables slanting at about $45^{\circ}$ : to be consistent with the identical 2-D images, the tables need to be of very different dimensions. 


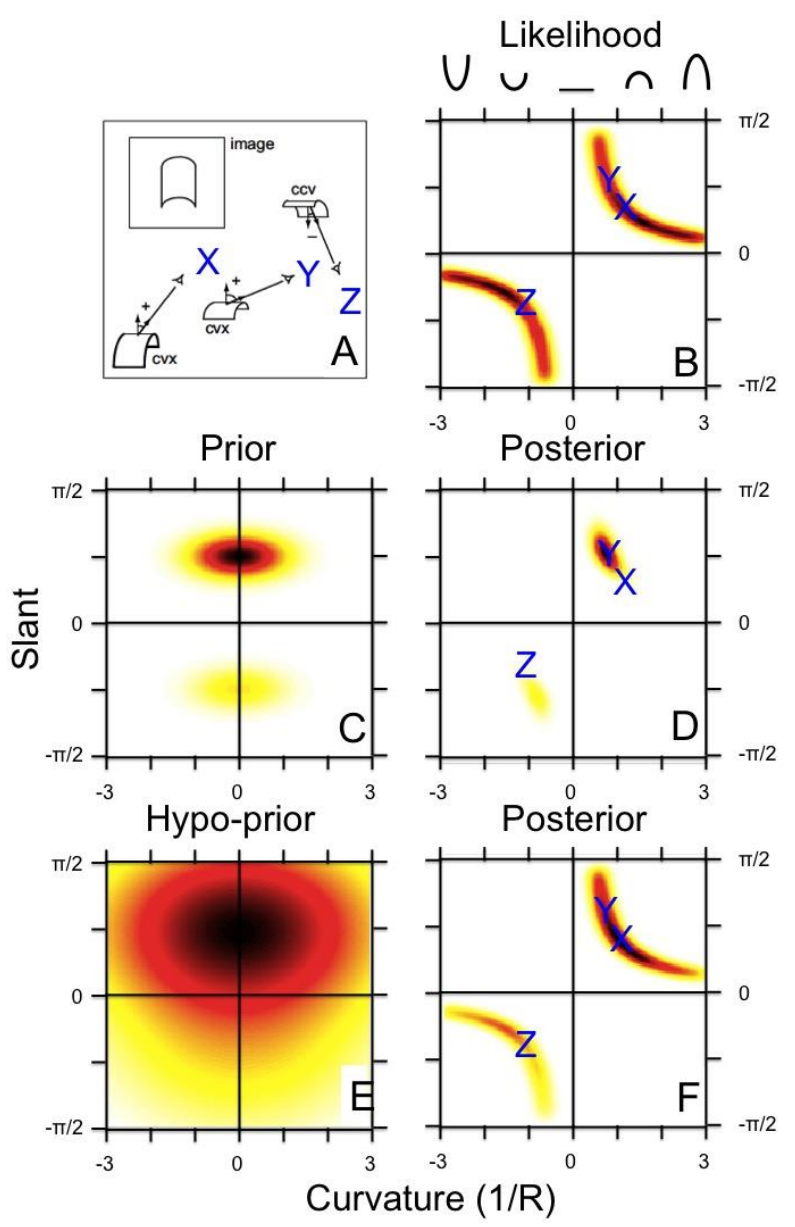

Figure 2. Illustration of how Bayesian priors can help resolve perceptual ambiguities (see Box I for more detailed explanation). A. An image (I) of a Tuscan Coppo tile, consistent with many physical shapes $(S)$, three of which are illustrated (X, Y \& Z). B. The likelihood function I|S shows the physical compatibilities of scene interpretations $S$ with image I, plotting curvature of the image (where I/R is a halfcircle) against image slant (where 0 represents a top view, $\Pi / 2$ end-on). $X, Y \& Z$ correspond to the examples in A. C. An estimate of a typical observer's prior, corresponding to a preference for low curvatures, and for perceiving objects from above. D. When the prior is multiplied by the likelihood to yield the posterior, the range of solutions is much more confined. The maximum of the posterior (MAP) is the best Bayesian estimate of the shape $S$ to generate image I (corresponding to Z). E. A prior like that in panel $\mathrm{C}$, except 10 times broader, as may be the case in autism. F. When this prior is multiplied by the likelihood, the posterior is far less constrained, and all three possibilities $X, Y$ $\& Z$ are possible. 


\section{Outstanding Questions}

- Is the in-built prior knowledge (prior) of people with autism attenuated compared to typically developing individuals of similar age and ability?

- Can people with autism modify prior knowledge - to the same extent as typically developing individuals? What are the effects of imposing an experimentally controlled prior [cf. 64]?

- Do individual differences in the strength of priors relate to differences in the degree of autistic symptoms (e.g., sensory sensitivities)?

- Can the idiosyncratic pattern of sensory atypicalities in an individual be accounted for by differences in exposure to particular environmental stimuli?

- Why is social information processing especially at risk in autism? Is it because social stimuli are inherently more complex and ambiguous than non-social stimuli?

- Is the way that people with autism view the world around them characterized by "Bayesian surprise" [65]?

- How can this account be related to Bayesian models of other neuropsychiatric conditions? For example, in schizophrenia, hallucinations are assumed to result from hyper-priors [66, 67], which would place autistic and schizophrenic symptoms at the extremes of a continuum. 


\section{References}

1. American, Psychiatric Association (2012) Proposed revision: Autism spectrum disorder. URL accessed 15th August 2012:

http://www.dsm5.org/proposedrevisions/pages/proposedrevision.aspx?rid=94.

2. Rogers, S. J. and Ozonoff, S. (2005) Annotation: what do we know about sensory dysfunction in autism? A critical review of the empirical evidence. J Child Psychol Psychiatry 46, 1255-1268.

3. Bogdashina, OI B. ga (2003) Sensory perceptual issues in autism and asperger syndrome : different sensory experiences - different perceptual worlds, Jessica Kingsley.

4. Williams, Donna (1994) Somebody somewhere : breaking free from the world of autism, Jessica Kingsley, 1999.

5. Bertone, A. et al. (2005) Enhanced and diminished visuo-spatial information processing in autism depends on stimulus complexity. Brain 128, 2430-2441.

6. Happe, F. and Frith, U. (2006) The weak coherence account: detail-focused cognitive style in autism spectrum disorders. J Autism Dev Disord 36, 5-25.

7. Mitchell, Peter and Ropar, Danielle (2004) Visuo-spatial abilities in autism: A review. Infant and Child Development 13, 185-198.

8. Mottron, L. et al. (2006) Enhanced perceptual functioning in autism: an update, and eight principles of autistic perception. J Autism Dev Disord 36, 27-43.

9. Plaisted, K. (2001) Reduced generalization in autism: An alternative to weak central coherence. In J. Burack, T. Charman, N. Yirmiya, \& P. Zelazo (Eds.), The development of autism : perspectives from theory and research, Erlbaum.

10. Baron-Cohen, S. et al. (2009) Talent in autism: hyper-systemizing, hyper-attention to detail and sensory hypersensitivity. Philos Trans R Soc Lond B Biol Sci 364, 1377-1383.

11. Shah, A. and Frith, U. (1983) An islet of ability in autistic children: a research note. J Child Psychol Psychiatry 24, 613-620.

12. Happe, F. G. (1996) Studying weak central coherence at low levels: children with autism do not succumb to visual illusions. A research note. J Child Psychol Psychiatry 37, 873-877. 
13. Miller, L. K. (1999) The savant syndrome: intellectual impairment and exceptional skill. Psychol Bull 125, 31-46.

14. Joseph, R. M. et al. (2009) Why is visual search superior in autism spectrum disorder? Dev Sci 12, 1083-1096.

15. Plaisted, K. et al. (1998a) Enhanced visual search for a conjunctive target in autism: a research note. J Child Psychol Psychiatry 39, 777-783.

16. Plaisted, K. et al. (1998b) Enhanced discrimination of novel, highly similar stimuli by adults with autism during a perceptual learning task. J Child Psychol Psychiatry 39, 765-775.

17. Simmons, D. R. et al. (2009) Vision in autism spectrum disorders. Vision Res 49, 2705-2739.

18. Franklin, A. et al. (2010) Reduced chromatic discrimination in children with autism spectrum disorders. Dev Sci 13, 188-200.

19. Bonnel, A. et al. (2003) Enhanced pitch sensitivity in individuals with autism: a signal detection analysis. J Cogn Neurosci 15, 226-235.

20. Pellicano, E. et al. (2005) Abnormal global processing along the dorsal visual pathway in autism: a possible mechanism for weak visuospatial coherence? Neuropsychologia 43, 1044-1053.

21. Behrmann, M. et al. (2006) Configural processing in autism and its relationship to face processing. Neuropsychologia 44, 110-129.

22. Dalton, K. M. et al. (2005) Gaze fixation and the neural circuitry of face processing in autism. Nat Neurosci 8, 519-526.

23. Pellicano, E. and Macrae, C. N. (2009) Mutual eye gaze facilitates person categorization for typically developing children, but not for children with autism. Psychon Bull Rev 16, 1094-1099.

24. Senju, A. et al. (2004) Reflexive orienting in response to eye gaze and an arrow in children with and without autism. J Child Psychol Psychiatry 45, 445-458.

25. Blake, R. et al. (2003) Visual recognition of biological motion is impaired in children with autism. Psychol Sci 14, 151-157.

26. Koldewyn, K. et al. (2010) The psychophysics of visual motion and global form processing in autism. Brain 133, 599-610. 
27. Kuhl, P. K. et al. (2005) Links between social and linguistic processing of speech in preschool children with autism: behavioral and electrophysiological measures. Dev Sci 8, F1-F12.

28. Mottron, L. et al. (2009) Enhanced perception in savant syndrome: patterns, structure and creativity. Philos Trans R Soc Lond B Biol Sci 364, 1385-1391.

29. Baron-Cohen, S. and Belmonte, M. K. (2005) Autism: a window onto the development of the social and the analytic brain. Annu Rev Neurosci 28, 109-126.

30. Rubenstein, J. L. and Merzenich, M. M. (2003) Model of autism: increased ratio of excitation/inhibition in key neural systems. Genes Brain Behav 2, 255-267.

31. Kanner, L (1943) Autistic disturbances of affective contact. Nervous Child 2, 217-250.

32. Helmholtz, Hermann von and Southall, James P. C. b (1962) Helmholtz's treatise on physiological optics. Translated from the 3d German ed. Edited by James P. C. Southall, New York, Dover Publications [1962].

33. Gregory, R. L. (1980) Perceptions as hypotheses. Philos Trans R Soc Lond B Biol Sci 290, 181-197.

34. Knill, D. C. and Pouget, A. (2004) The Bayesian brain: the role of uncertainty in neural coding and computation. Trends Neurosci 27, 712-719.

35. Kersten, D. et al. (2004) Object perception as Bayesian inference. Annu Rev Psychol 55, 271-304.

36. Kersten, D. and Yuille, A. (2003) Bayesian models of object perception. Curr Opin Neurobiol 13, 150-158.

37. Mamassian, P. et al. (2002) Bayesian modelling of visual perception. In R.P.N. et al. (eds). Probabilistic Models of the Brain: Perception and Neural Function.

38. Mamassian, P. et al. (1998) The perception of cast shadows. Trends Cogn Sci 2, 288-295.

39. Weiss, Y. et al. (2002) Motion illusions as optimal percepts. Nat Neurosci 5, 598-604.

40. Hollingworth, H.L. (1910) The central tendency of judgements. J. Philos Psychol Sci Methods 7, 461-469.

41. Jazayeri, M. and Shadlen, M. N. (2010) Temporal context calibrates interval timing. Nat Neurosci $13,1020-1026$.

42. Cicchini, G. M. et al. (2012) Optimal Encoding of Interval Timing in Expert Percussionists. Journal of Neuroscience 32, 1056-1060. 
43. Ma, W. J. et al. (2006) Bayesian inference with probabilistic population codes. Nat Neurosci 9, 1432-1438.

44. Fiser, J. et al. (2010) Statistically optimal perception and learning: from behavior to neural representations. Trends Cogn Sci 14, 119-130.

45. Cherkassky, V. L. et al. (2006) Functional connectivity in a baseline resting-state network in autism. Neuroreport 17, 1687-1690.

46. Greenaway, R. and Plaisted, K. (2005) Top-down attentional modulation in autistic spectrum disorders is stimulus-specific. Psychol Sci 16, 987-994.

47. Iarocci, G. et al. (2006) Global-local visual processing in high functioning children with autism: structural vs. implicit task biases. J Autism Dev Disord 36, 117-129.

48. Loth, E. et al. (2010) When seeing depends on knowing: adults with Autism Spectrum Conditions show diminished top-down processes in the visual perception of degraded faces but not degraded objects. Neuropsychologia 48, 1227-1236.

49. Soulieres, I. et al. (2010) Category induction in autism: slower, perhaps different, but certainly possible. Q J Exp Psychol (Hove) 64, 311-327.

50. Soulieres, I. et al. (2007) Atypical categorical perception in autism: autonomy of discrimination? J Autism Dev Disord 37, 481-490.

51. Mottron, L. et al. (1999) Local bias in autistic subjects as evidenced by graphic tasks: perceptual hierarchization or working memory deficit? J Child Psychol Psychiatry 40, 743-755.

52. Ropar, D. and Mitchell, P. (2002) Shape constancy in autism: the role of prior knowledge and perspective cues. J Child Psychol Psychiatry 43, 647-653.

53. Becchio, C. et al. (2010) Perception of shadows in children with autism spectrum disorders. PLoS One 5, e10582.

54. Kohn, A. (2007) Visual adaptation: physiology, mechanisms, and functional benefits. J Neurophysiol 97, 3155-3164.

55. Barlow, H. (1990) Conditions for versatile learning, Helmholtz's unconscious inference, and the task of perception. Vision Res 30, 1561-1571. 
56. Andrews, DP (1964) Error-correcting perceptual mechanisms. Quart. J. Exp. Psychol. 16, 104115.

57. Pellicano, E. et al. (2007) Abnormal adaptive face-coding mechanisms in children with autism spectrum disorder. Curr Biol 17, 1508-1512.

58. Tannan, V. et al. (2008) Perceptual metrics of individuals with autism provide evidence for disinhibition. Autism Res 1, 223-230.

59. Fiorentini, C. et al. (2011) Relatives of children with autism show diminished adaptation to facial identity. Manuscript in preparation.

60. Chopin, A. and Mamassian, P. (2012) Predictive properties of visual adaptation. Curr Biol 22, 622-626.

61. Stocker, A. A. and Simoncelli, E. P. (2006) Sensory adaptation within a Bayesian framework for perception. In Y. Weiss, B. Schoelkopf, \& J. Platt (Eds.). Advance in neural information processing systems.

62. Gregory, R. (1998) Brainy mind. Bmj 317, 1693-1695.

63. Grandin, T. (2009) Visual abilities and sensory differences in a person with autism. Biol Psychiatry 65, 15-16.

64. Kording, K. P. and Wolpert, D. M. (2004) Bayesian integration in sensorimotor learning. Nature 427, 244-247.

65. Baldi, P. and Itti, L. (2010) Of bits and wows: A Bayesian theory of surprise with applications to attention. Neural Netw 23, 649-666.

66. Corlett, P. R. et al. (2009) From drugs to deprivation: a Bayesian framework for understanding models of psychosis. Psychopharmacology (Berl) 206, 515-530.

67. Fletcher, P. C. and Frith, C. D. (2009) Perceiving is believing: a Bayesian approach to explaining the positive symptoms of schizophrenia. Nat Rev Neurosci 10, 48-58. 